\title{
Grupo de Ginástica Orientada na Terceira Idade: condições funcionais e adesão
}

\section{Group Oriented Gymnastics in Old Age: functional and accession conditions}

\author{
Grupo de Gimnasia Orientada en la Vejez: \\ condiciones funcionales $y$ adhesión
}

Recebido: 20/08/2013

Aprovado: $15 / 01 / 2014$

\author{
Mariana Colombini Buranello ${ }^{1}$ \\ Nayara Cândida Gomes ${ }^{2}$ \\ Lislei Jorge Patrizzi ${ }^{3}$ \\ Marta Regina Farinelli ${ }^{4}$ \\ Suraya Gomes Novais Shimano 5
}

Este é um estudo quantitativo realizado de 2011 a 2013 com o objetivo de caracterizar o grupo de ginástica orientada realizada por uma equipe multiprofissional quanto às condições funcionais e à adesão. Participaram do estudo 27 idosos do grupo "Saúde em Movimento" que foram submetidos à avaliação da pressão arterial, índice de massa corporal, circunferência da cintura, flexibilidade e equilíbrio. A adesão foi verificada nos arquivos de frequência. Os resultados demonstraram predominância do sexo feminino; idade de 70,4 anos; tempo no grupo de 23,6 meses; teste de equilíbrio e mobilidade de 10,8 segundos, apontando risco de queda em 56\% do grupo. A adesão foi de 6\% entre 2011 a 2013. Com base na avaliação, foi proposta uma intervenção de exercícios específicos para as necessidades do grupo, com frequência semanal de três vezes e duração de 60'. Observou-se que, dentre as condições físicas avaliadas, o equilíbrio corporal é um fator importante de risco para quedas, portanto a intervenção contínua e específica pode proporcionar melhora físico-funcional nesse grupo.

Descritores: Idoso, Promoção da Saúde, Ginástica.

This is a quantitative study carried through of 2011 the 2013 with aim to characterize the group fitness oriented performed by a multidisciplinary team, for the functional conditions and adherence. The study included 27 elderly group "Health in Motion" that were submitted to blood pressure, body mass index, waist circumference, flexibility and balance. Compliance was checked in files often. The results showed a predominance of females, aged 70.4 years, while in the group of 23.6 months, test balance and mobility 10.8 seconds indicating fall risk in $56 \%$ of the group. Adherence was $6 \%$ between 2011 and 2013. Based on the evaluation, it proposed an exercise intervention specific to the needs of the group, with three times weekly frequency and duration of 60 '. It was observed that among the physical conditions evaluated, body balance is an important risk factor for falls, therefore, continued intervention and can provide specific physical and functional improvement in this group.

Descriptors: Aged, Health Promotion, Gymnastics.

Esto es un estudio cuantitativo hecho de 2011 al 2013 con el objetivo de caracterizar el grupo de gimnasia orientada realizado por un equipo multidisciplinario, para las condiciones funcionales y la adherencia. Participaron 27 personas de edad avanzada de grupo "Salud en Movimiento" que se sometió a la verificación de la presión de la sangre, índice de masa corporal, circunferencia de la cintura, flexibilidad y el equilibrio. La adhesión se comprobó en los archivos de frecuencia. Los resultados mostraron un predominio del sexo femenino, con edades entre 70,4 años, mientras que en el grupo de 23,6 meses, el balance de la prueba y la movilidad 10,8 segundos que indica el riesgo de caídas en el $56 \%$ del grupo. La adhesión fue del $6 \%$ entre 2011 y 2013. Basándose en la evaluación fue propuesto intervención de ejercicios específicos para las necesidades del grupo, con frecuencia semanal de tres veces y duración de 60'. Se observó que, entre las condiciones físicas evaluadas, el equilibrio del cuerpo es un factor de riesgo importante para las caídas, por lo tanto, la intervención continúa puede proporcionar una mejora física y funcional específica en este grupo.

Descriptores: Anciano, Promoción de la Salud, Gimnasia.

\footnotetext{
${ }^{1}$ Fisioterapeuta. Especialista em Fisioterapia Geriátrica. Residente em Saúde do Idoso pela Universidade Federal do Triângulo Mineiro (UFTM). marianacolombinib@yahoo.com.br

2 Enfermeira. Residente em Saúde do Idoso pela UFTM. nayarinha gomes@hotmail.com

${ }^{3}$ Fisioterapeuta. Doutora em Clínica Médica. Professora Adjunta do Departamento de Fisioterapia Aplicada da UFTM. Ipatrizzi@uol.com.br.

${ }^{4}$ Assistente Social. Doutora em Serviço Social. Docente do Departamento de Serviço Social da UFTM. martaregina@servicosocial.uftm.edu.br.

5 Fisioterapeuta. Doutora em Ciências da Reabilitação. Docente do Departamento de Fisioterapia Aplicada da UFTM. surayagnovais@gmail.com.
} 


\section{INTRODUÇÃO}

$\mathrm{N}$ os últimos anos, no Brasil, pode-se observar o desenvolvimento sistemático de um processo de envelhecimento populacional. De acordo com o Instituto Brasileiro de Geografia e Estatística (IBGE), em 2009, $11,3 \%$ da população brasileira era composta por idosos, e estima-se que, no ano de 2050, este número ultrapasse os 64 milhões, representando mais de $22 \%$ da população ${ }^{1-3}$.

Este aumento evidencia a necessidade de intervenções que assegurem a integração progressiva e equilibrada dos idosos na sociedade, buscando preservar a qualidade, o significado e o direito à vida nas idades avançadas ${ }^{4}$.

Para que as pessoas possam vivenciar um envelhecimento bemsucedido, é fundamental construir a melhor relação possível entre quantidade e qualidade de vida. A Organização Mundial da Saúde (OMS) definiu o termo "envelhecimento ativo" como processo de otimização das oportunidades de saúde, participação e segurança, para expressar o decurso de conquista do envelhecimento como experiência positiva ${ }^{1}$.

0 indivíduo idoso consegue chegar aos oitenta ou noventa anos, mas não consegue evitar os efeitos do envelhecimento natural. Esses efeitos traduzem-se em alterações físicofuncionais como fraqueza muscular, alterações no equilíbrio e no balance $\mathrm{e}$ modificações na mobilidade, entre outras, levando a um aumento do risco de quedas, depressão e hospitalizações ${ }^{4}$.

O Sistema Único de Saúde (SUS) tem como princípios a Universalidade, a Integralidade e a Equidade, adotando um conceito ampliado, em que saúde é o estado de completo bem-estar físico, mental e social e não apenas a ausência de doença ${ }^{5}$.

Várias estratégias vêm sendo criadas para que esses princípios possam ser operacionalizados pelos serviços de saúde ${ }^{6}$. Esse é o caso das residências multiprofissionais em saúde, que vêm, por meio da formação dos profissionais nos princípios do SUS, problematizar o "[...] processo de trabalho e sua capacidade de dar acolhimento e cuidado às várias dimensões e necessidades de saúde das pessoas, dos coletivos e das populações"7.

Assim, a Residência Integrada Multiprofissional em Saúde (RIMS) - Área de Concentração em Saúde do Idoso, da Universidade Federal do Triângulo Mineiro (UFTM), tem como objetivo desenvolver competências fundamentadas nas diretrizes do SUS para exercer ações específicas de acordo com o núcleo de saber e prática da área profissional (Serviço Social, Enfermagem, Fisioterapia, Nutrição, Educação Física e Terapia Ocupacional, Biomedicina, Educação Física), nos diferentes campos de saberes e práticas previstas e planejar as intervenções direcionadas a indivíduos, família e coletividade, considerando o perfil epidemiológico da população, os princípios do SUS e a atuação em equipes multiprofissionais.

O Projeto de Extensão "Saúde em Movimento para a Terceira Idade" consiste de um grupo de ginástica orientada realizada em uma Unidade Básica de Saúde (UBS) de Uberaba-MG, e busca promover a prática regular de exercício físico planejado, supervisionado e executado por profissionais capacitados, voltada à comunidade e com ênfase nos idosos.

Estudos demonstram que os exercícios físicos possuem papel importante na vida de pessoas idosas, bem como ressaltam que estes, quando praticados de forma sistemática, somados a outros hábitos saudáveis como alimentação, lazer e cultura, aumentam a expectativa de vida bem como a qualidade desta. Certamente, para que o exercício físico regular tenha os efeitos esperados, a aplicação de estímulos deve ser adequada, sendo supervisionada por um profissional ${ }^{4}$.

Dentro desse contexto, este estudo tem como objetivo caracterizar o Grupo de Ginástica Orientada realizada por uma 
Equipe Multiprofissional quanto às condições funcionais e à adesão.

\section{MÉTODO}

Trata-se de um estudo de caráter transversal, quantitativo e descritivo. As avaliações e intervenções estão amparadas pelo projeto de pesquisa aprovado pelo Comitê de Ética em Pesquisa da UFTM sob protocolo n. ${ }^{\circ}$ 2189/2011.

Os sujeitos selecionados foram os idosos participantes do projeto de extensão "Saúde em Movimento para a Terceira Idade", com idade acima de 60 anos, que frequentavam o grupo de ginástica pelo menos duas vezes por semana e aceitaram participar da avaliação por meio da assinatura do Termo de Consentimento Livre e Esclarecido.

As intervenções de promoção da saúde ocorrem no projeto de extensão de ginástica orientada há três anos, numa UBS de Uberaba - MG, cenário de prática dos residentes em Saúde do Idoso da RIMS UFTM. Este projeto busca promover a prática regular de exercício físico planejado, supervisionado e executado por profissionais capacitados, voltado para a comunidade idosa. A frequência é registrada desde o primeiro ano de atuação, o que possibilita a análise da adesão ao grupo. Para ser classificado como assíduo no grupo, o idoso deveria estar presente pelo menos duas vezes durante a semana.

A periodização do grupo é de três vezes por semana, com duração de 60 minutos e aferição da pressão arterial antecedendo a realização dos exercícios. Os exercícios propostos são baseados nos resultados da avaliação e constam de um período de aquecimento (10'), alongamento (10'), exercícios resistidos, treino de equilíbrio e coordenação (30') e relaxamento (10').

Para a avaliação das condições físicas dos idosos, foi proposta a mensuração das seguintes variáveis:

- $\quad$ Sinais vitais e antropometria: Pressão arterial - colocou-se o indivíduo com o braço apoiado no nível do coração, com cinco minutos de repouso, selecionando-se o tamanho da braçadeira de acordo com indivíduo. A largura do manguito correspondeu a $40 \%$ da circunferência braquial e seu comprimento a $80 \%$. Localizou-se a artéria braquial ao longo da face interna superior do braço, palpando-a. Manteve-se a margem anterior da braçadeira $2,5 \mathrm{~cm}$ acima da dobra do cotovelo. Determinou-se o nível máximo de insuflação palpando o pulso radial até seu desaparecimento, registrando o valor (pressão sistólica palpada) e aumentando mais $30 \mathrm{mmHg}$. Posicionou-se o estetoscópio $\mathrm{BD} \AA$ sobre a artéria braquial palpada abaixo do manguito na fossa anticubital, em seguida desinsuflou-se o manguito de modo que a pressão caísse de 2 a $3 \mathrm{mmHg}$ por segundo, identificando-se a pressão sistólica e diastólica em milímetros de mercúrio ${ }^{8}$.

- Circunferência da cintura - os indivíduos permaneceram na posição ortostática com o abdômen relaxado, a fita ficou posicionada no plano horizontal no nível natural da cintura, que é a parte mais estreita do tronco no ponto médio entre a última costela e a crista ilíaca. A leitura foi feita no momento da expiração ${ }^{8}$.

- Peso e altura - o indivíduo foi posicionado em cima de uma balança digital da marca Phillips $\AA^{\circledR}$ com precisão de $100 \mathrm{~g}$, sem os sapatos e com vestimenta leve. A altura foi medida com um estatiômetro próprio da balança, com precisão de $1 \mathrm{~mm}$, utilizandose o método da estatura alongada. A equação IMC $=$ peso/altura ${ }^{2}$ foi utilizada para a determinação do índice de massa corpórea (IMC) ${ }^{9}$;

- Flexibilidade com a utilização do Banco de Wells (Instant Flex, Sanny®, Brasi). Para a realização do teste, o avaliado sentou-se em frente à caixa, apoiou os pés no local indicado e manteve os joelhos estendidos. Solicitou-se que o indivíduo levasse os braços estendidos à frente da régua localizada no centro do banco, sem flexionar os joelhos. 0 resultado escolhido foi o melhor de três tentativas em um intervalo de 30 segundos; 
- Equilíbrio e mobilidade, através do teste Timed Up and Go (TUG) ${ }^{10}$. Com o idoso sentado em uma cadeira sem apoio para os braços, foi solicitado a este que levantasse da cadeira sem a ajuda dos braços, andasse uma distância de três metros, desse a volta e retornasse. No início do teste, o indivíduo estava com o dorso apoiado no encosto da cadeira e, ao final, encostava novamente. 0 indivíduo recebeu a instrução "vá" para realizar o teste, e o tempo foi cronometrado a partir da voz de comando até o momento em que ele apoiou novamente o dorso no encosto da cadeira. A realização do teste em até 10 segundos é considerada normal para idosos da comunidade, sendo um tempo maior que esse considerado um preditor de quedas ${ }^{11}$.

Os resultados da avaliação física foram descritos em termos de frequência para variáveis categóricas, e de médias e desvio padrão para as variáveis numéricas com base no programa estatístico SPSS $®$ (Statistical Package for Social Sciences) versão 19.

\section{RESULTADOS}

Participaram do projeto "Saúde em Movimento para a Terceira Idade" 36 pessoas, sendo que, destas, cinco tinham menos de 60 anos e quatro idosos não estavam classificados como assíduos no grupo, totalizando 27 idosos inclusos no estudo. A idade média foi de 70,4 $( \pm 6,4)$ anos, houve predominância do sexo feminino com $85,2 \%$ e tempo no grupo de $23,6( \pm 20,8)$ meses. Observou-se grande variabilidade no tempo de grupo, o que demonstra alto índice de adesão de novos membros ao grupo.

A Tabela 1 demonstra os resultados da avaliação física. É possível observar que a média da pressão arterial sistólica foi de $123,7( \pm 13) \mathrm{mmHg}$ e diastólica 72,2 $( \pm 6,4)$ $\mathrm{mmHg}$. Em relação ao IMC, foi de 25,6 ( \pm 3$)$ $\mathrm{Kg} / \mathrm{m}^{2}$, e a circunferência da cintura de $90,1( \pm 9,9) \mathrm{cm}$. Em relação ao equilíbrio e à mobilidade, foi de 10,8 $( \pm 2,9)$ segundos e $18,5( \pm 10,5) \mathrm{cm}$ para flexibilidade.
O Gráfico 1 mostra a classificação do risco para quedas de acordo com o teste TUG, sendo que $56 \%$ dos participantes do grupo foram classificados com risco para quedas.

Tabela 1. Avaliação física de idosos pesquisados. Uberaba, 2013.

\begin{tabular}{lrr}
\hline Variável & Média & \multicolumn{1}{c}{ DP* } \\
\hline Pressão Arterial Sistólica (mmHg) & 123,7 & 13,0 \\
Pressão Arterial Diastólica (mmHg) & 72,2 & 6,4 \\
Circunferência da Cintura (cm) & 90,1 & 9,9 \\
Índice de Massa Corporal $\left(\mathrm{Kg} / \mathrm{m}^{2}\right)$ & 25,6 & 3,0 \\
Flexibilidade $(\mathrm{cm})$ & 18,5 & 10,5 \\
Teste TUG (s.) & 10,8 & 2,9 \\
\hline
\end{tabular}

Gráfico 1. Risco para quedas de acordo com o teste TUG. Uberaba, 2013.

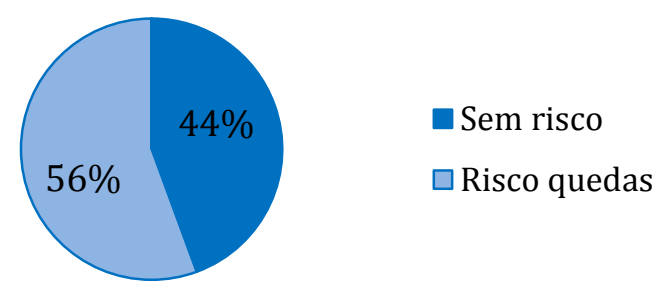

0 Gráfico 2 demonstra a adesão ao grupo durante os três anos. Pode-se observar a adesão crescente, sendo que, do ano de 2011 até o ano de 2013 houve aumento de quase $6 \%$ no número médio de participantes.

Gráfico 2. Adesão ao projeto - média e desvio padrão. Uberaba, 2013.

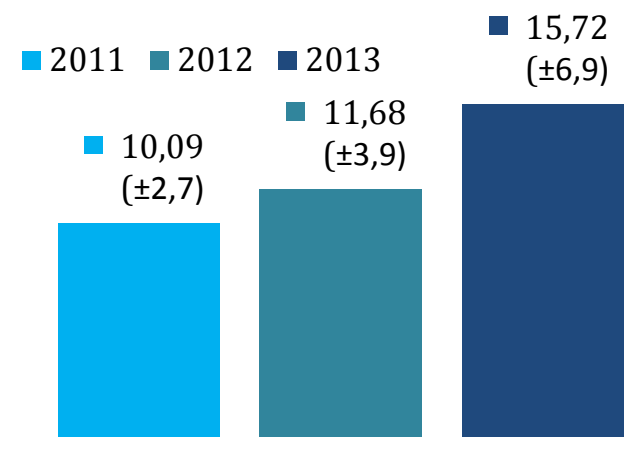

\section{DISCUSSÃO}

Os resultados apontam predominância do sexo feminino estando de acordo com outros estudos da qual há maior participação de mulheres na prática de 
atividades físicas. A maior participação feminina é atribuída a aspectos culturais, religiosidade, aspectos motivacionais, maior cuidado com a saúde, criação de vínculos e o relacionamento entre as idosas nas atividades ${ }^{12,13}$.

A avaliação física evidenciou valor médio de IMC considerado eutrófico para idosos, de acordo com os valores propostos por Lipschitz ${ }^{14}$. Os valores de circunferência da cintura foram considerados limítrofes, podendo indicar um risco latente para eventos cardiovasculares ${ }^{15}$. Já a pressão arterial sistólica e diastólica apresentou média nos valores de normalidade de acordo com as diretrizes vigentes ${ }^{8}$.

A avaliação física evidenciou pontos importantes a serem trabalhados na prescrição de atividades, como exercícios de alongamento da musculatura de membros inferiores e o treino de equilíbrio e propriocepção, objetivando a redução do risco de quedas, tal como preconizado em outros estudos com grupos de idosos ${ }^{16-19}$. Os achados da avaliação física estão de acordo com outras pesquisas, que aponta a diminuição da flexibilidade ${ }^{20,21}$ e alterações nos sistemas de controle do equilíbrio postural decorrentes do envelhecimento ${ }^{22,23}$, levando o idoso a estar mais propenso a sofrer queda ${ }^{24}$. Esses episódios de quedas podem ocasionar redução de autoestima, da autonomia, esgotamento físico e mental, ansiedade e o medo de novas quedas ${ }^{25}$. Além disso, em decorrência destas, maiores são os índices de hospitalização, a reabilitação é mais demorada, aumenta-se o risco de dependência e morte ${ }^{26}$. Ressalta-se que o índice de quedas em idosos no Brasil é extremamente alto, sendo considerado problema de saúde pública ${ }^{27}$.

A habilidade de evitar quedas, em qualquer situação, depende inteiramente da extensão na qual a perturbação desafia a capacidade de estabilização postural. Seu risco aumentado pode resultar da habilidade reduzida do sistema de equilíbrio em se recuperar de perturbações, mesmo que mínimas ${ }^{28}$, o que justifica a necessidade de intervenção nesse sistema durante a prática de exercício em grupo.

Os valores de desvio padrão das variáveis físicas como pressão arterial, circunferência da cintura e flexibilidade e do tempo de permanência no grupo foram expressivos. Isso dificultou a discussão dos resultados junto com a de outros estudos com grupos de ginástica orientada já publicados. Desta forma, a diversidade de tempo da prática na atividade física dos idosos do grupo refletiu-se na variabilidade de resultados na avaliação física, que se apresentaram de forma heterogênea.

Houve adesão crescente ao grupo de ginástica orientada coordenada pela equipe de residência multiprofissional em saúde do idoso. Isto demonstra a importância da equipe multiprofissional no cuidado a essa população. Em três anos, diversas ações de divulgação e estímulo à prática de atividades físicas foram realizadas. Dentre elas, se pode citar o "Dia do Idoso", em que folders com dicas para prevenção de quedas e exercícios foram distribuídos aos idosos, e o "Dia da Fisioterapia", em que houve a prática de ginástica orientada com os usuários da UBS pela qual, os residentes atuam, bem como, se divulgou no Programa HiperDia.

Pensando nas políticas públicas relacionadas ao idoso, a Política Nacional de Saúde da Pessoa Idosa ${ }^{28}$, em especial, estabelece como uma de suas diretrizes a promoção do envelhecimento ativo e saudável. Essa diretriz objetiva o envelhecer mantendo a capacidade funcional e a autonomia, sendo reconhecidamente a meta de toda ação de saúde. Os profissionais de saúde e a comunidade devem perceber que a prevenção de agravos e a promoção da saúde não são privilégios apenas dos jovens. Ou seja, a promoção não termina quando se faz 60 anos, e as ações de prevenção devem ser incorporadas à atenção à saúde em todas as idades.

A diretriz seguinte diz respeito à atenção integral e integrada ao idoso e à importância do ambiente nas quais estão 
inseridos, estabelecendo que a prática de cuidados às pessoas idosas exige abordagem global, interdisciplinar e multidimensional, que leve em conta a grande interação entre os fatores físicos, psicológicos e sociais que influenciam a saúde dos idosos. 0 trabalho interdisciplinar se faz essencial ao cuidado do idoso, sendo os programas de residência multiprofissionais um recurso eficaz para a efetivação dessa política ${ }^{29}$.

A Política Nacional de Promoção da Saúde tem por objetivo geral promover a qualidade de vida e reduzir vulnerabilidade e riscos à saúde relacionados aos seus determinantes e condicionantes - modos de viver, condições de trabalho, habitação, ambiente, educação, lazer, cultura, acesso a bens e serviços essenciais. Dentre as suas ações específicas, está a prática corporal e de atividade física. Essa ação busca ofertar práticas corporais e atividades físicas como caminhadas, prescrição de exercícios, práticas lúdicas, esportivas e de lazer na Atenção Primária à Saúde, voltadas tanto para a comunidade como um todo quanto para grupos vulneráveis, como a população idosa. Além disso, a política de promoção da saúde também prevê ações de avaliação dessas práticas por meio do desenvolvimento de estudos e da formulação de metodologias capazes de produzir evidências e comprovar a efetividade de estratégias de práticas corporais/atividades físicas no controle e na prevenção das doenças crônicas não transmissíveis ${ }^{27}$.

\section{CONCLUSÃO}

0 presente estudo vai ao encontro do que estabelece as políticas públicas atuais, contribuindo para a promoção da saúde e a prevenção de complicações, em especial da pessoa idosa.

Observou-se que, dentre as condições físicas avaliadas, as alterações do equilíbrio corporal são um fator importante de risco para quedas, portanto, a intervenção contínua e específica pode proporcionar melhora físico-funcional para esse grupo.

\section{REFERÊNCIAS}

1. Organização Mundial da Saúde. Envelhecimento ativo: uma política de saúde. Brasília: Organização Pan-Americana da Saúde; 2005.

2. Instituto Brasileiro de Geografia e Estatística. Síntese de indicadores sociais: uma análise das condições de vida da população brasileira 2010 [Internet]. Rio de Janeiro: IBGE; 2010. [acesso em 24 jul 2013]. Disponível

em: http://www.ibge.gov.br/home/estatistica/p opulacao/condicaodevida/indicadoresminim os/sinteseindicsociais2010/SIS_2010.pdf.

3. Instituto Brasileiro de Geografia e Estatística. Censo Demográfico 2010: características da população e dos domicílios: resultados do universo. Rio de Janeiro: IBGE; 2011.

4. Farinatti PTV. Envelhecimento, Promoção da Saúde e Exercício: bases teóricas e metodológicas. São Paulo: Manole; 2008.

5. Pinheiro R, Ceccim RB, Mattos RA, orgs. Ensinar saúde: a integralidade e o SUS nos cursos de graduação na área da saúde. Rio de Janeiro: IMS/UERJ: ABRASCO; 2005.

6. Brasil. Lei no 8080, de 19 de setembro de 1990. Dispõe sobre as condições para a promoção, proteção e recuperação da saúde, a organização e o funcionamento dos serviços correspondentes e dá outras providências [Internet]. D.O.U. Brasília, DF, 20 set 1990 [acesso em 06 agosto 2013]. Disponível em: http://portal.saude.gov.br/portal/arquivos/ pdf/LEI8080.pdf.

7. Ceccim RB, Feuerwerker LCM. O Quadrilátero da Formação para a Área da Saúde: ensino, gestão, atenção e controle social. Physis 2004; 14(1):41-65.

8. Sociedade Brasileira de Cardiologia. Departamento de Cardiogeriatria. II Diretrizes em Cardiogeriatria da Sociedade Brasileira de Cardiologia. Arq Bras Cardiol. 2010; 95(3 supl.2):1-112.

9. Norton K, Olds T. Antropometria. Porto Alegre: Artmed; 2005. 
10. Podsiadlo D, Richardson S. The timed "Up \& Go": a test of basic functional mobility for frail elderly pearsons. J Am Geriatr Soc. 1991; 39(2):142-8.

11. Bischoff HA, Stähelin HB, Monsch AU, Iversen MD, Weyh $\mathrm{A}$, Von Dechend $\mathrm{M}$ et al. Identifying a cut-off point for normal mobility: a comparison of the timed 'up and go' test in community-dwelling and institutionalised elderly women. Age Ageing. 2003; 32(3):315-20.

12. Nunes FRT, Duarte G. A preponderância do gênero feminino nas atividades físicas para o idoso na Secretaria Municipal de Esportes de Porto Alegre. Rev Bras Ciênc Envelhec Hum. 2011; 8(2):230-43.

13. Queiroz Junior CA, Castellano SM, Paulo TRS, Moreira WW, Simões R. Motivos de adesão das mulheres idosas participantes dos programas públicos de exercícios físicos em Uberaba-MG. Colec Pesq Educ Fis. 2012; 11(4):135-42.

14. Lipschitz DA. Screening for nutritional status in the elderly. Prim Care. 1994; 21(1):55-67.

15. World Health Organization. Obesity: preventing and managing the global epidemic: report of a Who Consultation (WHO Technical Report Series, 894). Geneva: WHO; 2000. Defining the problem of overweight and obesity; p. 241-3.

16. Paulo TRS, Castellano SM. Queiroz Junior CA, Freitas Junior IF. Programa Academia da Terceira Idade no município de Uberaba, MG. Arq Ciênc Esporte. 2012; 1(1):54-9.

17. Resende SM, Rassi CM, Viana FP. Efeitos da hidroterapia na recuperação do equilíbrio e prevenção de quedas em idosas Braz J Phys Ther, 2008; 12(1):57-63.

18. Rebelatto JR, Calvo JI, Orejuela JR, Portillo JC. Influência de um programa de atividade física de longa duração sobre a força muscular manual e a flexibilidade corporal de mulheres idosas. Braz J Phys Ther. 2006; 10(1):127-32.

19. Pellegrinotti IL, Silva FV. Comparação da preensão manual e da flexibilidade de mulheres acima de 50 anos praticantes de atividade física com índices de referência para saúde. HU Rev. 2011; 37(4):487-94.

20. Silva NA, Menezes TN, Melo RLP, Pedraza DF. Força de preensão manual e flexibilidade e suas relações com variáveis antropométricas em idosos. Rev Assoc Med Bras. 2013; 59(2):128-35.

21. Ruwer LS, Rossi AG, Simon LF. Equilíbrio no idoso. Rev Bras Otorrinolaringol. 2005; 71(3):298-303.

22. Almeida APPV, Veras RP, Doimo LA. Avaliação do equilíbrio estático e dinâmico de idosas praticantes de hidroginástica e ginástica. Rev Bras Cineantropom Desempenho Hum. 2010; 12(1):55-61.

23. Meneses SRF, Burke TN, Marques AP. Equilíbrio, controle postural e força muscular em idosas osteoporóticas com e sem quedas. Fisioter Pesq. 2012; 19(1):26-31.

24. Lopes KT, Costa DF, Santos LF, Castro DP, Bastone AC. Prevalência do medo de cair em uma população de idosos da comunidade e sua correlação com mobilidade, equilíbrio dinâmico, risco e histórico de quedas. Braz J Phys Ther. 2009; 13(3):223-9.

25. Melo EG, Azevedo E. Quedas no idoso. Temas Rematol Clin. 2007; 8(4):121-7.

26. Mazo GZ et al. Condições de saúde, incidência de quedas e nível de atividade física dos idosos. Braz J Phys Ther. 2007; 11(6):437-42.

27. Centro de Estudos Ortopédicos do HSPE. Manual de Prevenção de Quedas da Pessoa Idosa [Internet]. São Paulo: IAMSPE; [200-?]. [acesso em 20 fev 2013]. Disponível em http://www.iamspe.sp.gov.br/imagens/Carti lhasManuais/CartilhaQuedaIdoso11-04.pdf . 28. Ministério da Saúde (Br). Portaria GM no 2.528, de 19 de outubro de 2006 [Internet]. Aprova a Política Nacional de Saúde da Pessoa Idosa - PNSI. D.O.U. Brasília, DF, 20 out. 2006. [acesso em 22 fev. 2013]. Disponível em: http://pesquisa.in.gov.br/imprensa/jsp/visu aliza/index.jsp?jornal $=1 \&$ pagina $=142 \&$ data $=$ 20/10/2006

29. Ministério da Saúde (Br). Secretaria de Vigilância em Saúde. Política Nacional de Promoção da Saúde. Brasília: Ministério da Saúde; 2006. 60 p.

\footnotetext{
CONTRIBUIÇÕES

Mariana Colombini Buranello e Nayara Candida Gomes contribuíram na aplicação da avaliação e na intervenção com o grupo,
} 
na análise dos resultados e na redação do artigo;

Lislei Jorge Patrizzi, Marta Regina

Farinelli e Suraya Gomes Novais

Shimano atuaram no planejamento, delineamento do estudo e revisão crítica do artigo. 